\title{
Transcriptome analysis deciphers the mechanisms of exogenous nitric oxide action on the response of melon leaves to chilling stress
}

\author{
Q. DIAO ${ }^{1}, \mathrm{Y}_{\mathrm{CAO}} \mathrm{CH}^{1}$ H. FAN ${ }^{2 *}$, and Y. ZHANG ${ }^{1 *}$ \\ Horticultural Research Institute and Shanghai Key Laboratory of Protected Horticultural Technology, \\ Shanghai Academy of Agricultural Sciences, Shanghai 201403, P.R. China ${ }^{1}$ \\ Shanghai Agriculture Technology Extension and Service Center, Shanghai 201103, P.R. China
}

\begin{abstract}
Chilling stress is a major abiotic factor that limits the growth and productivity of melon (Cucumis melo L.). The application of nitric oxide (NO) can enhance plant tolerance to chilling stress; however, the underlying molecular mechanisms for this process remain poorly understood. In this study, RNA sequencing was performed on melon seedlings exposed to control conditions, chilling stress, or chilling stress in the presence of NO donor sodium nitroprusside (SNP), to identify NO-mediated transcript changes in response to chilling stress. The results identified 488, 1012 , and 1589 differentially expressed genes (DEGs) between plants in optimum conditions (CK) and chilling stress (CS) groups, plants in the CS and chilling stress + SNP $(\mathrm{CN})$ groups, and those in CK and CN groups, respectively. Through gene ontology $(G O)$ database and Kyoto encyclopedia of genes and genomes (KEGG) enrichment analyses, the DEGs were classified as predominantly involved in saccharide metabolism, biosynthesis of other secondary metabolites, lipid metabolism, amino-acid metabolism, and signal transduction pathways. In addition, 39 genes related to sugar metabolism including those encoding UDPglucuronate-4-epimerase, $\beta$-glucosidase, glucuronosyltransferase, $\alpha$-1,4-galacturonosyl transferase, and hexokinase, were upregulated in the CK vs. CS comparison, and genes encoding fructose-bisphosphate aldolase and glucan-endo-1,3- $\beta$ glucosidase were upregulated in the CS vs. CN, and CK vs. CN comparisons. A gene encoding an EREBP-like factor was upregulated in the CK vs. CS, CS vs. CN, and CK vs. CN comparisons. The expression profiles of 10 selected genes were analyzed using real-time quantitative PCR, and the candidate gene expression patterns were consistent with the DEG classification from RNA-seq. Overall, the data provide insight into the transcriptional regulation by exogenous NO in the response of melon seedlings to chilling stress. The data from this study are relevant for further research on the molecular mechanisms that underlie chilling resistance in melon plants.
\end{abstract}

Additional key words: Cucumis melo, differentially expressed genes, RNA sequencing, sodium nitropruside, sugar metabolism.

\section{Introduction}

Melon is one of the most economically important and commonly consumed fruit crops in many countries, but it is sensitive to and often suffers damage from chilling stress, especially in the winter or early spring. Chilling stress is a common environmental stress that can affect crop growth and agricultural productivity, since it can induce physiological, biochemical and molecular responses (Sitnicka and Orzechowski 2014). Thus, it is extremely important to enhance the chilling tolerance of melon seedlings.

Chilling tolerance can be increased in plants by cold acclimation, which results in multiple reprogramming mechanisms, including changes in gene expression and biochemical and physiological modifications (Zhu et al. 2007). Evidence suggests that nitric oxide (NO) production plays a critical role in chilling tolerance (Cantrel et al. 2011, Zhao et al. 2011). Nitric oxide is a ubiquitous reactive signaling molecule that is involved in diverse developmental and physiological processes, and in various plant responses to abiotic stresses, such as drought, salt, temperature, and heavy metals (Siddiqui et al. 2011, Shi et al. 2012, 2014). NO participates in plant responses to abiotic stresses most probably by inducing antioxidant defense and by reducing the generation of reactive oxygen species (ROS) (Filippou et al. 2013, Sun et al. 2015). Recent studies have reported that NO treatment can

Submitted 25 October 2019, last revision 31 January 2020, accepted 5 February 2020.

Abbreviations: CK - controls; CN - chilling stress + nitric oxide; CS - chilling-stress; DEG - differentially expressed gene; GO - gene ontology; KEGG - Kyoto encyclopedia of genes and genomes; SNP - sodium nitroprusside.

Acknowledgements: This work was financially supported by the Shanghai Agricultural System Youth Talent Growth Plan Project (2018, 1-13) and by the Shanghai Fruit and Vegetable Industry Technology System [Shanghai Agricultural Science (2019) No. 1].

* Corresponding authors; e-mails: hongweifannjzx@163.com, zyp123944@126.com 
enhance plant stress resistance by influencing polyamine biosynthesis, chlorophyll content, and photosystem II activity (Chen et al. 2013, Fan et al. 2013). Furthermore, NO regulates genes that are involved in plant defense, oxidative stress responses, and plant hormone signaling (Lindermayr et al. 2005, Zago et al. 2006, Zeng et al. 2014, Hu et al. 2015, Li et al. 2019). Jian et al. (2016) and Rizwan et al. (2018) reported that the application of NO donor sodium nitroprusside (SNP) enhanced the expression of CAT, $A P X$, and $S O D$ in Medicago truncatula under salt stress and in rice under Ni-stress. Although numerous studies have identified NO-responsive genes, related information concerning the molecular mechanisms of NO-induced chilling tolerance in melon plants is lacking and it is crucial to improving melon breeding.

Currently, due to the availability of the complete melon genome sequence and high-throughput RNA sequencing (RNA-seq) technologies, a lot of genomic data concerning melon fruit development and quality have been generated (Garcia-Mas et al. 2012, Zhang et al. 2016, Shin et al. 2017). Yano et al. (2017) examined the transcriptome in 30 different tissues at 7 stages of melon fruit development and identified 20752 genes expressed in at least one tissue. In addition, RNA-seq data have revealed responsive genes or key pathways resulting from abiotic stress in melon. For example, Wang et al. (2016) identified the gene expression profiles in two muskmelon cultivars in response to salt stress. Genes responding to chilling stress and their corresponding pathways have been reported for other plant species, including cassava (An et al. 2012), rice (Zhang et al. 2012), watermelon (Xu et al. 2016), and Populus simonii (Song et al. 2013). However, to our knowledge, few transcriptome analyses have been reported on the response of melon to chilling stress. Because of the importance of melon as a crop, it is necessary to study NO-regulated and chilling stress-responsive gene networks in this species; therefore, this study investigated the transcriptional responses of melon to NO and chilling stress by RNA-seq analysis. The results represent a basis for improving its tolerance to chilling stress.

\section{Materials and methods}

Seeds of melon (Cucumis melo L.) cv. XL-1 were produced by our laboratory at Shanghai Academy of Agricultural Science, Shanghai, China, rinsed thoroughly with distilled water, and germinated in an incubator at a temperature of $30{ }^{\circ} \mathrm{C}$. The germinated seedlings were then transferred to plastic plates containing Vermiculite and grown in a growth chamber at day/night temperatures of $30 / 20{ }^{\circ} \mathrm{C}$, a 12-h photoperiod, an irradiance of $400 \mu \mathrm{mol} \mathrm{m} \mathrm{m}^{-2} \mathrm{~s}^{-1}$, and a relative humidity of $80 \%$. Plants were watered every $2 \mathrm{~d}$ with a 1/8-strength Hoagland solution (Hoagland and Snyder 1933). When melon seedlings reached the second true-leaf stage, the leaves were sprayed daily with $200 \mathrm{~cm}^{3}$ distilled water or $200 \mu \mathrm{M}$ sodium nitroprusside (SNP) for $3 \mathrm{~d}$. On the fourth day, the plants were exposed to chilling stress $\left(6^{\circ} \mathrm{C}\right.$ for $\left.3 \mathrm{~h}\right)$, the leaves of each group were harvested, immediately frozen in liquid nitrogen, and stored at $-70{ }^{\circ} \mathrm{C}$ for further analyses. Seedlings without treatments were used as the control. All experiments consisted of three replicates.

Total RNA was extracted using a mirVana miRNA isolation kit (Ambion, Austin, TX, USA) following the manufacturer's protocol. The RNA integrity was evaluated using an Agilent 2100 bioanalyzer (Agilent Technologies, Santa Clara, CA, USA). The samples with an RNA integrity number (RIN) $\geq 7$ were used for subsequent analyses. The cDNA libraries were constructed using the TruSeq Stranded mRNA LT Sample Prep kit (Illumina, San Diego, CA, USA) according to the manufacturer's instructions. The libraries were sequenced on the Illumina sequencing platform (HiSeqTM 2500 or Illumina HiSeq X $T e n$ ), and $125 \mathrm{bp} / 150 \mathrm{bp}$ paired-end reads were generated. Three biological replicates were performed for RNA-seq.

Raw reads were processed by removing reads containing adapters, reads containing poly- $\mathrm{N}$ and low-quality reads, to obtain clean data (clean reads). The remaining highquality clean reads were aligned to the melon reference genome sequences using HISAT software (Cock et al. 2009). The mapped reads were then reconstructed using Cufflink (Conesa et al. 2005). All RNA-seq reads were deposited at NCBI sequence read archive (SRA) database under the accession number PRJNA553119.

Gene expressions were calculated for each sample using RSEM software (McKenna et al. 2010). The differentially expressed genes (DEGs) among the three samples were identified using DESeq. The threshold of false discovery rate $\leq 0.05$ and an absolute fold change value $\geq 2$ were used as the threshold for DEG selection.

Gene ontology $(G O)$ enrichment analysis was performed using agriGO (Du et al. 2010); the Kyoto encyclopedia of genes and genomes (KEGG) pathway enrichment analysis was implemented by the $K E G G$ orthology-based annotation system (KOBAS) (Xie et al. 2011). Significantly enriched pathways with respect to DEGs were identified according to the criterion of a corrected $P$-value $\leq 0.05$.

Total RNA was extracted from the same samples with three biological replicates as those subjected to Illumina RNA-seq using the mirVanaTM RNA isolation kit, according to the manufacturer's specifications. Total RNA $(2 \mu \mathrm{g})$ from each sample was used to synthesize cDNA by $M-M L V$ (TaKaRa, Dalian, China). Real-time PCR was performed using LightCycler ${ }^{\circledR} 480$ real-time PCR instrument (Roche, Basel, Switzerland). The expressions of mRNAs were normalized to that of the reference gene Actin and were calculated using the $2^{-\Delta \Delta \mathrm{Ct}}$ method (Livak and Schmittgen 2001). Reactions were performed in a GeneAmp ${ }^{\circledR} 9700$ PCR system (Applied Biosystems, Foster City, CA, USA). Primers used for all real-time quantitative PCR experiments are listed in Table 1 Suppl.

\section{Results}

In this study, high-throughput transcriptomic sequencing technology was used to investigate the molecular mechanisms that regulate the effect of NO on the response of Cucumis melo to chilling stress. Samples exposed to 
chilling alone $(\mathrm{CS})$, chilling in the presence of SNP $(\mathrm{CN})$, or controls $(\mathrm{CK})$ were sequenced. In total, approximately 412 million raw reads were generated using the Illumina HiSeq TM 2500 platform. After removing adapters, reads

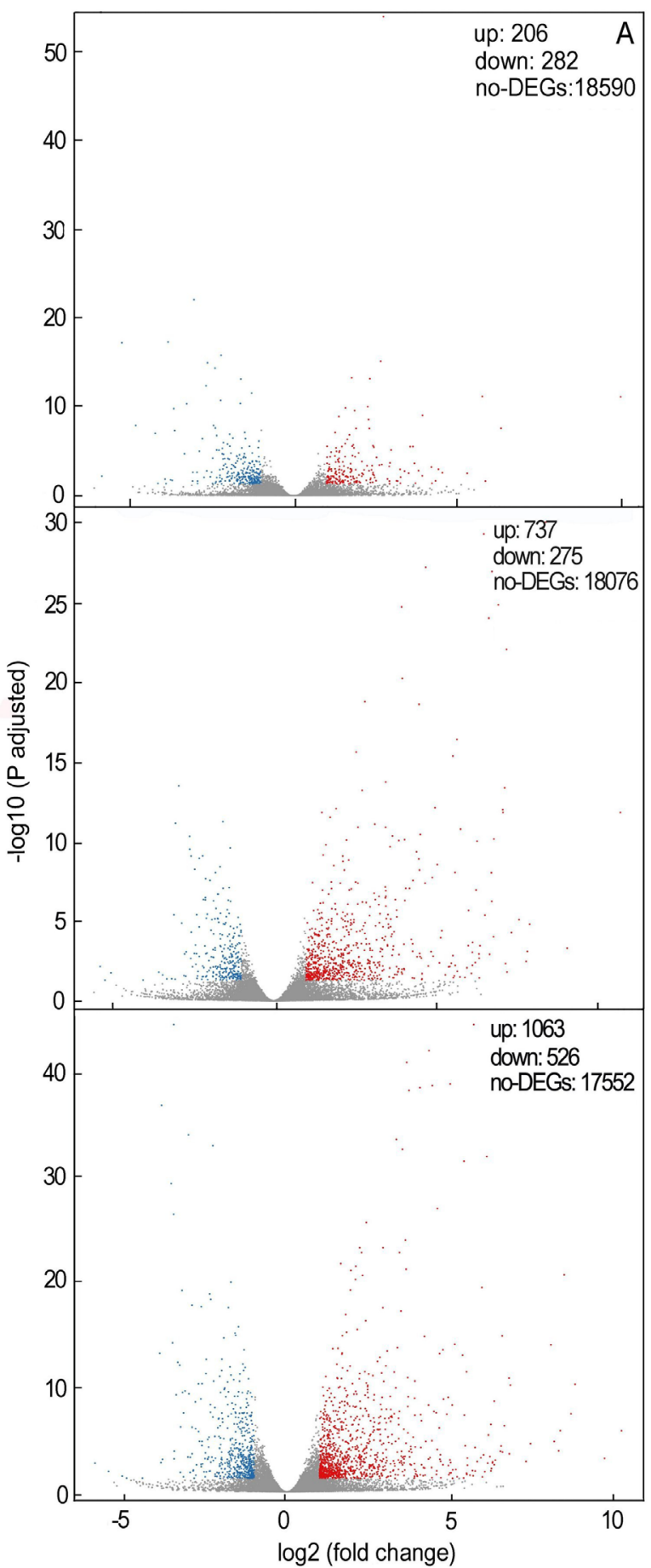

containing poly-N, and low-quality reads, approximately 379 million clean reads were obtained, representing $91.99 \%$ of the total raw reads (Table 2 Suppl.). The mean proportion of bases with mass no less than 20 or 30 after
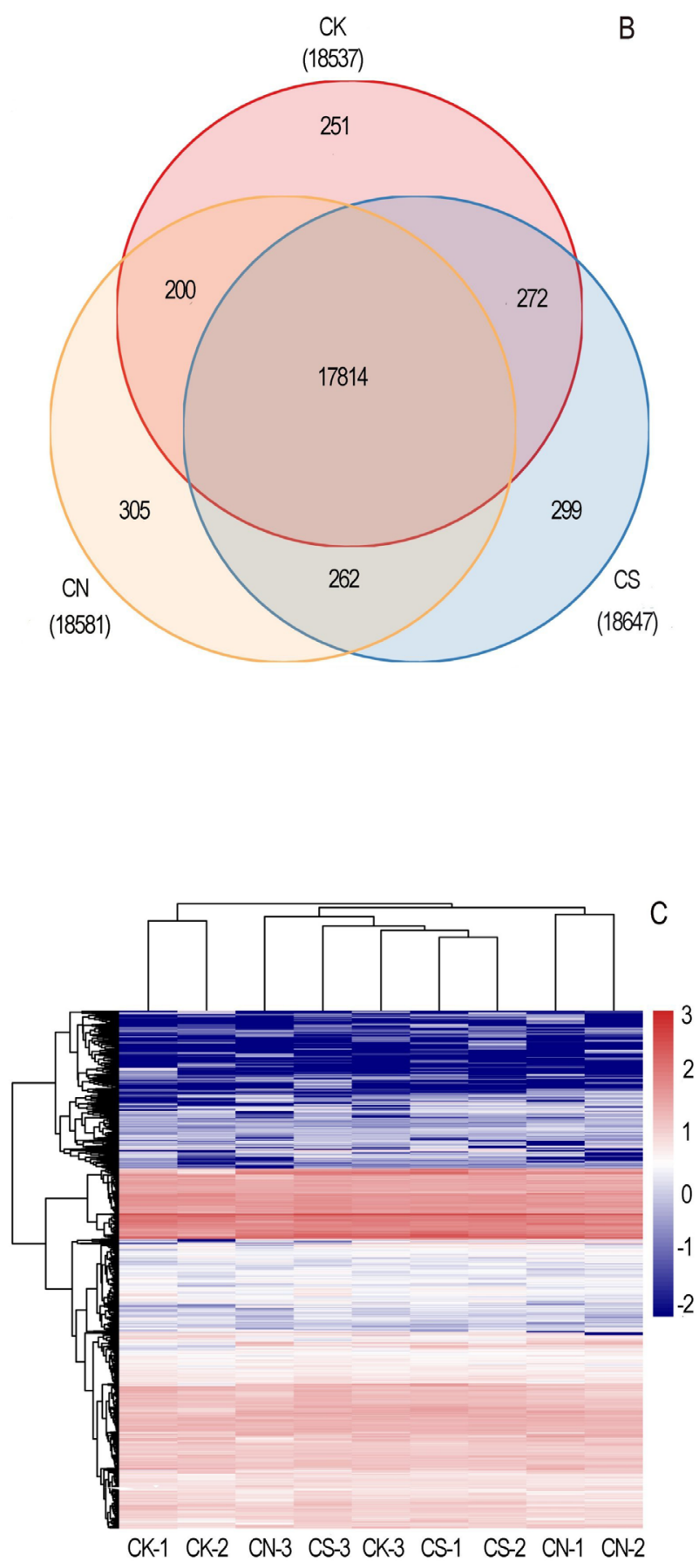

Fig. 1. Differentially expressed genes (DEGs) between samples. $A$ - The Volcano plot of DEGs. The $x$-axis represents the fold-change of DEGs in different experimental groups, and the $y$-axis represents the $P$ value $\left(\log _{\bullet}\right)$. Dots represent genes; the red dots represent upregulated DEGs and the blue dots represent downregulated DEGs. $B$ - The Venn diagram of DEGs. Total DEGs for each comparison are shown in parentheses. $C$ - The heat map of DEGs. Each row represents the $\log _{2}$ value of the expression of a gene in different treatments. Different gene expressions are displayed as a gradient ramp. A greater intensity of red indicates a higher gene expression; increasing blue intensity represents a decreasing gene expression. CK - control samples; CS - samples exposed to chilling stress; $\mathrm{CN}$ - samples exposed to chilling stress and $200 \mathrm{mM}$ sodium nitroprusside. Each treatment consisted of three biological duplicates. 


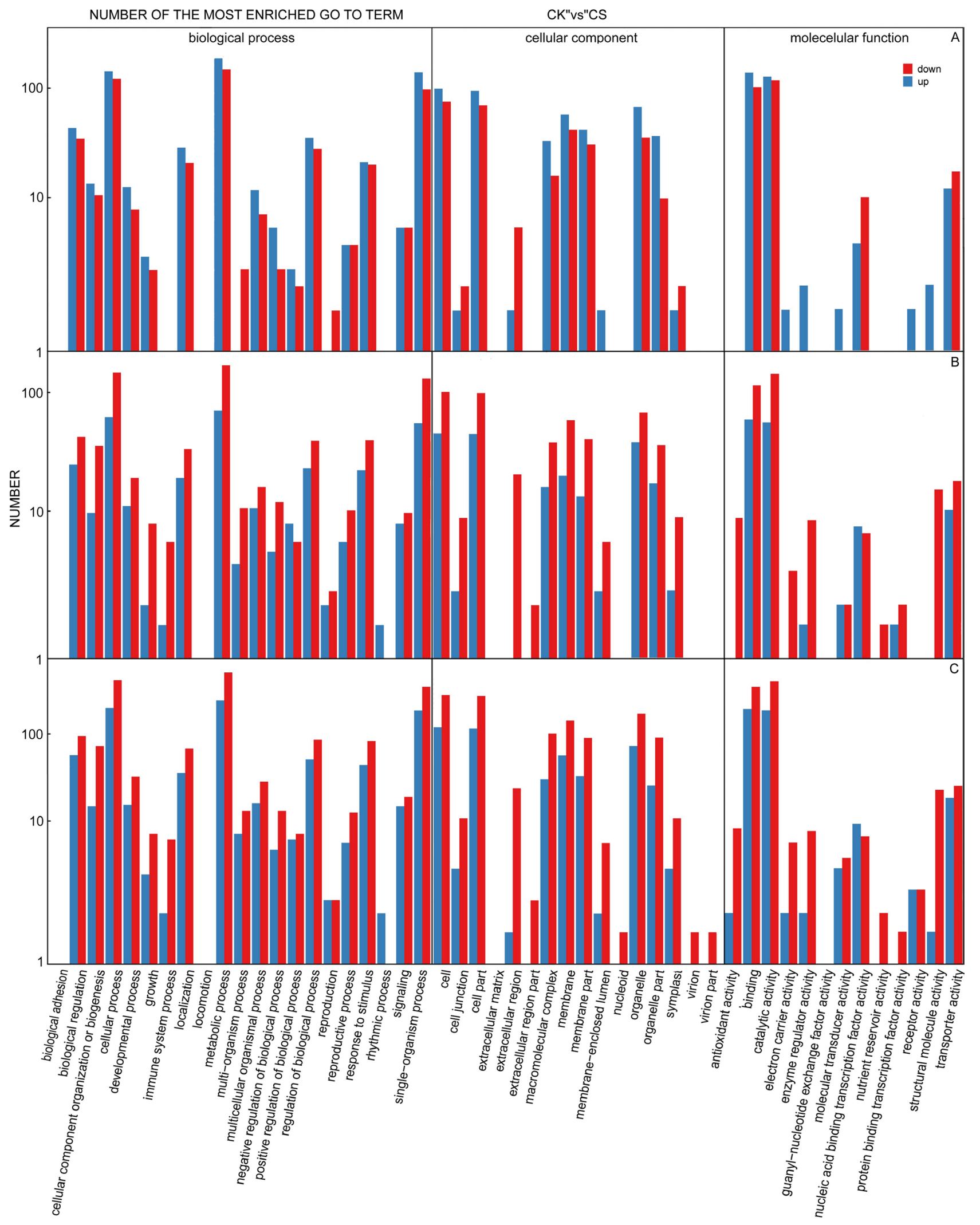

Fig. 2. Gene ontology $(G O)$ enrichment analysis of differentially expressed genes in different treatments. $A$ - Comparative $G O$ terms between control (CK) and chilling stress (CS) treatment; $B$ - comparative $G O$ terms between CS and chilling stress $+200 \mathrm{mM} \mathrm{SNP}$ $(\mathrm{CN})$ treatment; $C$ - comparative $G O$ terms between $\mathrm{CK}$ and $\mathrm{CN}$. The $x$-axis displays different $G O$ terms and the $y$-axis indicates the number of DEGs. 

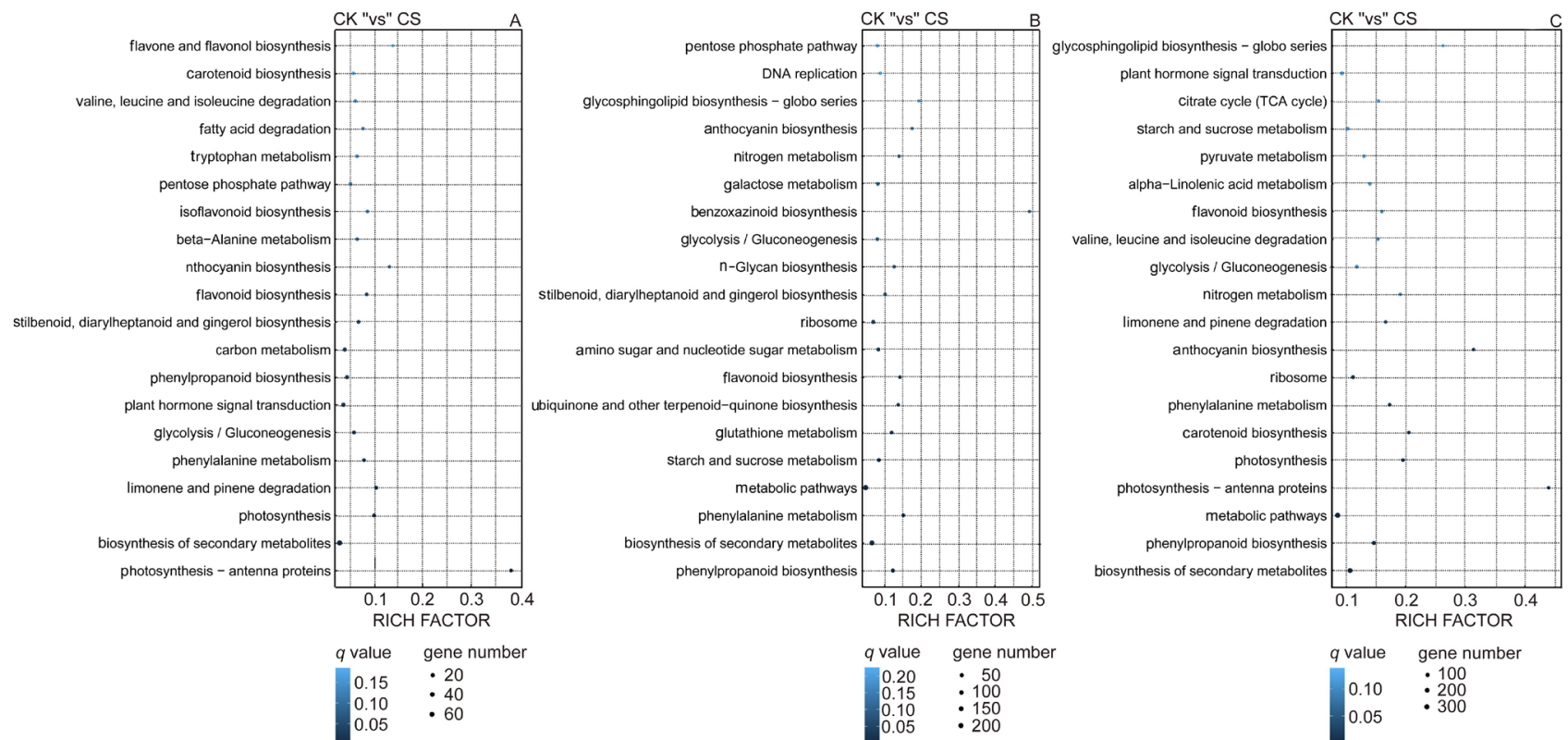

Fig. 3. Kyoto encyclopedia of genes and genomes enrichment analysis for different treatments. $A$ - differentially expressed genes (DEGs) in control (CK) vs. chilling stress (CS) treatments; $B$ - DEGs in CS vs. chilling stress $+200 \mathrm{mM} \mathrm{SNP}(\mathrm{CN})$ treatments; $C$ - CK vs. $\mathrm{N}$ teratments. The $x$-axis represents the rich factor and the $y$-axis represents pathways. Different colors of the dots correspond to the $q$ value range. The size of the dot represents the number of DEGs clustered in each pathway: larger dots indicate more DEGs. The rich factor indicates the degree of DEG enrichment in each pathway, the greater the rich factor, the greater the DEG enrichment.

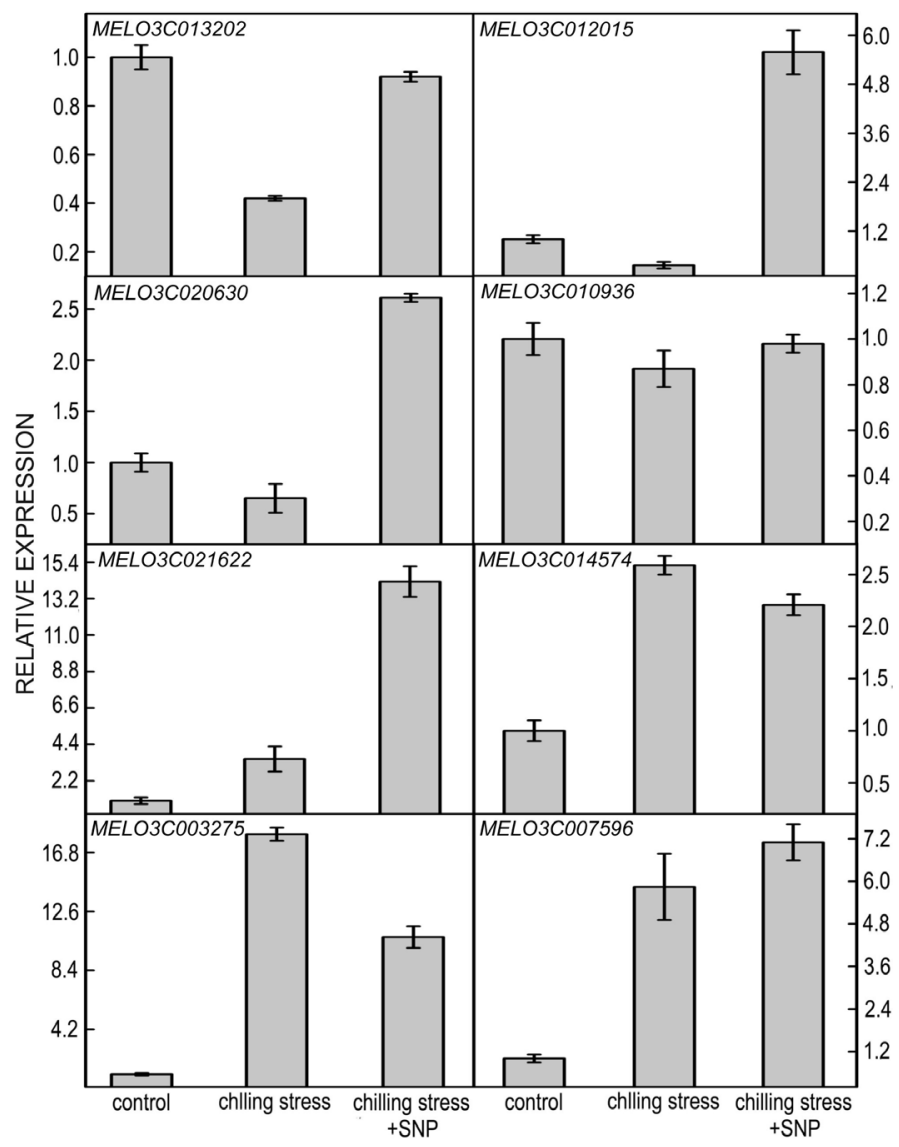

Fig. 4. Qantitative real time PCR reveals the transcripts of eight candidate genes under control, chilling stress, and chilling stress + sodium nitroprusside (SNP). 
filtration (Q20, Q30, respectively), and GC content were 96.66, 92.06, and 44.25\%, respectively (Table 3 Suppl.). The high-quality reads were then mapped to the $C$. melo L. genome and the mapped clean reads of the three data sets ranged from 78.89 to $89.52 \%$. Among these reads, 78.05 to $88.42 \%$ were uniquely mapped and were used for further analysis. In the control group vs. chilling stress treatment comparison, 488 DEGs were detected, with 206 DEGs being upregulated and 282 DEGs downregulated. The number of DEGs between the CS group and the CN group was 1589 , of which 1063 were upregulated and 526 were downregulated. Between the $\mathrm{CK}$ and $\mathrm{CN}$ groups, 1012 DEGs were identified, of which 737 were upregulated and 275 were downregulated (Fig. 1).

To further characterize the DEGs, they were functionally annotated using the $G O$ and $K E G G$ databases. The $G O$ functional enrichment analysis showed that all DEGs could be classified into 45 functional groups, including 20 groups in biological process, 13 in cellular component, and 12 in molecular function (Fig. 2). Within the 'biological process' category, 'metabolic process' and 'cellular process' were predominant. In the 'cellular components category', 'cell', 'cell part', and 'organelle' were predominant and 'binding', and 'catalytic activity' were the most common subcategories in the 'molecular function' category. In the CK vs. CS comparison, 'cell' (148) and 'cell part' (141) were the dominant 'cellular components' subcategories. The 'binding' (189) and 'catalytic activity' (192) were the most predominant 'molecular functions' subcategories. The main 'biological processes' subcategories were 'metabolic process' (249) and 'cellular process' (209) (Fig. 2A). In the CS vs. CN comparison, DEGs were primarily involved in 'cellular process', 'metabolic process' and 'single organism process' in the 'biological process' category, and the DEGs were enriched for $G O$ terms that included 'cell part', 'cell', 'binding', and 'catalytic activity' (Fig. 2B). As concern the $\mathrm{CK}$ and $\mathrm{CN}$ data, the $G O$-term distribution was similar to the first two groups described above (Fig. 2C).

To understand the biological function of genes further, we analyzed the DEGs using the KEGG database. In this study, $K E G G$ revealed that the pathways most highly represented were 'carbohydrate metabolism', 'biosynthesis of other secondary metabolites', followed by 'amino acid metabolism', 'lipid metabolism' and 'signal transduction'. This suggests that these pathways might be important in protecting melon plants against chilling stress (Fig. 3). In the CK vs. CS comparison, 'biosynthesis of secondary metabolites', 'carbon metabolism', 'phenylpropanoid biosynthesis', and 'plant hormone signal transduction' were predominantly enriched (Fig. $3 A$ ). In the CS vs. CN comparison, most DEGs were enriched in three pathways: 'metabolic pathways', 'biosynthesis of secondary metabolites', and 'phenylpropanoid biosynthesis' (Fig. 3B). Moreover, 'metabolic pathways', 'biosynthesis of secondary metabolites', and 'phenylpropanoid biosynthesis' were the main three pathways for DEGs in the CK vs. CN comparison (Fig. 3C).

In this study, changes in the expression of transcripts encoding enzymes related to sugar metabolism pathways were identified, such as $\beta$-glucosidase, UDP-glucuronate 4-epimerase, glucose-6-phosphate isomerase, triose phosphate isomerase, and hexokinase (Fig. 4). Comparing transcriptome data for control and chilling-stressed plants, most of these genes were downregulated. The expression of genes encoding fructose-bisphosphate aldolase, glucan endo-1,3- $\beta$-glucosidase, and raffinose synthase were upregulated in the CS vs. CN comparison. In the CK vs. CN comparison, DEGs encoding fructose-bisphosphate aldolase, glucan endo-1,3- $\beta$-glucosidase and UDPglucuronate 4-epimerase were also upregulated.

In addition to an activation of genes involved in sugar metabolism, an enrichment in transcripts associated with hormone signalling was observed. These DEGs included genes that encoded EREBP-like factors, WRKY transcription factors, an auxin-responsive GH3 protein, an auxin-responsive IAA protein, gibberellin 2-oxidase, an abscisic acid receptor PYR/PYL family protein, a myb proto-oncogene protein, and abscisic acid $8^{\prime}$-hydroxylase, which were all upregulated in the CK vs. CS group (Table 3 Suppl.).

\section{Discussion}

Sugars are important regulators of plant growth, not only as carbon and energy source but also as signaling molecules (Rosa et al. 2009, O'Hara et al. 2013, Ruan 2014). Furthermore, increasing numbers of studies have demonstrated that sugars can regulate photosynthesis, lipid metabolism, osmotic homeostasis, and gene expression in response to various abiotic stresses (Keunen et al. 2013, Sami et al. 2016). Genes associated with sugar metabolism are regulated differently by cold acclimation in tea plants, whereby $C s B A M, C s I N V 5, C s R S 2$ are upregulated and CsSWEET2, 3, 16, CsERD6.7 and CsINT2 are downregulated (Yue et al. 2015). Therefore, varying content of sugars and activities of related metabolic pathways are crucially important to confer tolerance against diverse abiotic stresses. In this study, sugar metabolism was predominantly enriched upon chilling stress in melon leaves (CK vs. CS), including 16 upregulated genes (Fig. 3). Among these, four genes (encoding UDP-glucuronate 4-epimerase, $\beta$-glucosidase, glucuronosyltransferase, and $\alpha$-1,4-galacturonosyl transferase) were upregulated in response to chilling stress. Glucose and fructose are monosaccharides and can be phosphorylated by hexokinase (HXK) and/or fructokinase (FRK) during glycolysis, to provide substrates for numerous physiological pathways. In this study, CmHXK was upregulated and 6-phosphate fructokinase was downregulated by chilling stress. Genes encoding UDP-glucuronate 4-epimerase, fructosebisphosphate aldolase, glucan endo-1,3- $\beta$-glucosidase were upregulated by combined chilling stress and SNP treatment (CS vs. CN). Uridine diphosphate glucose dehydrogenase (UGD) catalyzes oxidation of UDPglucose to UDP-glucuronate, which is then converted to UDP-galacturonic acid, UDP-xylose, UDP-arabinose, and UDP-apiose (Seifert 2004). These sugars are precursors for the synthesis of polymers such as hemicellulose and 
pectin in plant cell walls. Therefore, we propose that the induced metabolism of sugars in response to SNP potentially provides an osmoprotectant function against membrane injury, which thereby contributes to chilling tolerance in melon seedlings.

Phytohormones, including ABA, SA, and auxin, are involved in plant adaptation to stress (Gan 2010, Davies 2010). The data here indicate that many genes related to hormone pathways, including those encoding abscisic acid 8'-hydroxylase, a PYR/PYL family member, an auxin responsive $\mathrm{GH} 3$ family member, and gibberellin 2-oxidase were upregulated during chilling stress (Table 3 Suppl.). Consistent with previous studies, GH3 and auxinrelated gene families have been reported to be involved in responses to biotic and abiotic stresses in rice (Hagen and Guilfoyle 2002). Many studies have demonstrated that transcription factor networks regulate abiotic stress responses (Chen et al. 2012). In this study, genes encoding an EREBP-like factor, WRKY transcription factor 2 and a myb proto-oncogene protein were significantly upregulated after exposure to chilling stress. Taken together, our results suggest that hormones might play crucial roles in plant chilling stress responses.

\section{Conclusions}

In this study, we performed RNA-seq to comparatively analyze the transcriptome of melon seedlings exposed to chilling stress or chilling stress combined with NO treatment. A large number of DEGs were identified in response to NO treatment under chilling stress. The results indicate that exogenous NO upregulates many genes related to sugar metabolism, including UDP-glucuronate 4-epimerase, $\beta$-glucosidase, glucuronosyltransferase, $\alpha$-1,4-galacturonosyl transferase, as well as $E R F / E R E B P$, WRKY, GH3 and PYR/PYL genes, which are involved in diverse signaling pathways. A significant positive correlation between qPCR results and the RNA-seq data for 10 genes confirmed the reliability of the transcriptome data. Collectively, these results provide an important contribution to the current understanding NO mediation of the chilling tolerance of melon seedlings but the specific regulatory mechanisms require further study.

\section{References}

An, D., Yang, J., Zhang, P.: Transcriptome profiling of low temperature-treated cassava apical shoots showed dynamic responses of tropical plant to cold stress. - BMC Genomics 13: 64. 2012.

Cantrel, C., Vazquez, T., Puyaubert, J., Rezé, N., Lesch, M., Kaiser, W.M., Baudouin, E.: Nitric oxide participates in cold-responsive phosphosphingolipid formation and gene expression in Arabidopsis thaliana. - New Phytol. 189: 415427, 2011.

Chen, K., Chen, L., Fan, J., Fu, J.: Alleviation of heat damage to photosystem II by nitric oxide in tall fescue. - Photosynth. Res. 116: 21-31, 2013.
Chen, L.G., Song, Y., Li, S.J., Zhang, L.P., Zou, C.S., Yu, D.Q.: The role of WRKY transcription factors in plant abiotic stresses. - Biochim. biophys. Acta 1819: 120-128, 2012.

Cock, P.J., Fields, C.J., Goto, N., Heuer, M.L., Rice, P.M.: The Sanger FASTQ file format for sequences with quality scores, and the Solexa/Illumina FASTQ variants. - Nucl. Acids Res. 38: $1767-1771,2009$

Conesa, A., Götz, S., García-Gómez, J.M., Terol, J., Talón, M., Robles, M.: Blast2GO: a universal tool for annotation, visualization and analysis in functional genomics research. Bioinformatics 21: 3674-3676, 2005.

Davies, P.J.: The plant hormones: their nature, occurrence, and functions. - In: Davies, P.J. (ed.): Plant Hormones: Biosynthesis, Signal Transduction, Action. Pp. 1-15. Springer, Dordrecht - Ithaca - New York 2010.

Du, Z., Zhou, X., Ling, Y., Zhang, Z., Su, Z.: agriGO: a GO analysis toolkit for the agricultural community. - Nucl. Acids Res. 38: W64-W70, 2010.

Fan, H.F., Du, C.X., Guo, S.R.: Nitric oxide enhances salt tolerance in cucumber seedlings by regulating free polyamine content. - Environ. exp. Bot. 86: 52-59. 2013.

Filippou, P., Antoniou, C., Fotopoulos, V.: The nitric oxide donor sodium nitro-prusside regulates polyamine and proline metabolism in leaves of Medicago truncatula plants. - Free Radicals Biol. Med. 56: 172-183, 2013.

Gan, S.: The hormonal regulation of senescence. - In: Davies, P.J. (ed.): Plant Hormones: Biosynthesis, Signal Transduction, Action. Pp. 597-617. Springer, Dordrecht - Ithaca - New York 2010.

Garcia-Mas, J., Benjak, A., Sanseverino, W., Bourgeois, M., Mir, G., González, V. M., Alioto, T.: The genome of melon (Cucumis melo L.). - Proc. nat. Acad. Sci. USA 109: 1187211877, 2012.

Hagen, G., Guilfoyle, T.J.: Auxin-responsive gene expression: genes, promoters and regulatory factors. - Plant mol. Biol. 49: 373-385, 2002.

Hoagland, D.R., Snyder, W.C.: Nutrition of strawberry plants under controlled conditions: (a) Effects of deficiencies of boron and certain other elements; (b) Susceptibility to injury from sodium salts. - Proc. amer. Soc. hort. Sci. 30: 288-294, 1933.

Hu, X., Yang, J., Li, C.: Transcriptomic response to nitric oxide treatment in Larix olgensis Henry. - Int. J. mol. Sci. 16: 28582-28597, 2015.

Keunen, E., Peshev, D., Vangronsveld, J., Ende,W., Cuypers, A.: Plant sugars are crucial players in the oxidative challenge during abiotic stress: extending the traditional concept. - Plant Cell Environ. 36: 1242-1255, 2013.

Jian, W., Zhang, D.W., Zhu, F., Wang, S.X., Pu, X.J., Deng, X.G., Lin, H.H.: Alternative oxidase pathway is involved in the exogenous SNP-elevated tolerance of Medicago truncatula to salt stress. - J. Plant Physiol. 193: 79-87, 2016.

Lindermayr, C., Saalbach, G., Durner, J.: Proteomic identification of S-nitrosylated proteins in Arabidopsis. - Plant Physiol. 137: 921-930, 2005.

Li, S.W., Leng, Y., Shi, R.F.: Transcriptome characterization of gene profiling during early stage of nitric oxide-induced adventitious rooting in mung bean seedlings. - J. Plant Growth Regul. 39: 1-26, 2019.

Livak, K.J., Schmittgen, T.D.: Analysis of relative gene expression data using real-time quantitative PCR and the $2^{-\Delta C T}$ method. - Methods 25: 402-408, 2001.

McKenna, A., Hanna, M., Banks, E., Sivachenko, A., Cibulskis, K., Kernytsky, A., DePristo, M.A.: The genome analysis Toolkit: a MapReduce framework for analyzing nextgeneration DNA sequencing data. - Genome Res. 20: 1297 - 
1303, 2010.

O'Hara, L., Paul, M., Wingler, A.: How do sugars regulate plant growth and development? New insight in to the role of trehalose-6-phosphate. - Mol. Plants 6: 261-274, 2013.

Rizwan, M., Mostofa, M.G., Ahmad, M.Z., Imtiaz, M., Mehmood, S., Adeel, M., Tu, S.: Nitric oxide induces rice tolerance to excessive nickel by regulating nickel uptake, reactive oxygen species detoxification and defense-related gene expression. Chemosphere 191: 23-35, 2018.

Rosa, M., Prado, C., Podazza, G., Interdonato, R., Gonzalez, J.A., Hilal, M., Prado, F.E.: Soluble sugars- metabolism, sensing and abiotic stress. - Plant Signal Behav. 4: 388-393, 2009.

Ruan, Y.L.: Sucrose metabolism: gateway to diverse carbon use and sugar signaling. - Annu. Rev. Plant Biol. 65: 33-67, 2014.

Sami, F., Yusuf, M., Faizan, M., Faraz, A., Hayat, S.: Role of sugars under abiotic stress. - Plant Physiol. Biochem. 109: 5461,2016

Seifert, G. J.: Nucleotide sugar interconversions and cell wall biosynthesis: how to bring the inside to the outside. - Curr. Opin. Plant Biol. 7: 277-284, 2004.

Shi, H., Ye, T., Zhu, J. K., Chan, Z.: Constitutive production of nitric oxide leads to enhanced drought stress resistance and extensive transcriptional reprogramming in Arabidopsis. - J. exp. Bot. 65: 4119-4131, 2014.

Shi, H.T., Li, R.J., Cai, W., Liu, W., Fu, Z.W., Lu, Y.T.: In vivo role of nitric oxide in plant response to abiotic and biotic stress. - Plant Signal Behav. 7: 438-440, 2012.

Shin, A.Y., Kim, Y.M., Koo, N., Lee, S.M., Nahm, S., Kwon, S.Y.: Transcriptome analysis of the oriental melon (Cucumis melo L. var. makuwa) during fruit development. - Peer J. 5: e2834, 2017.

Siddiqui, M.H., Al-Whaibi, M.H., Basalah, M.O.: Role of nitric oxide in tolerance of plants to abiotic stress. - Protoplasma 248: 447-455, 2011.

Sitnicka, D., Orzechowski, S.: Cold-induced starch degradation in potato leaves - intercultivar differences in the gene expression and activity of key enzymes. - Biol. Plant. 58: 659-666, 2014

Song, Y., Chen, Q., Ci, D., Zhang, D.: Transcriptome profiling reveals differential transcript abundance in response to chilling stress in populus simonii. - Plant Cell Rep. 32: 14071425, 2013.

Sun, C., Liu, L., Yu, Y., Liu, W., Lu, L., Jin, C.: Nitric oxide alleviates aluminum induced oxidative damage through regulating the ascorbate-glutathione cycle in roots of wheat. J. integr. Plant Biol. 57: 550-561, 2015.

Wang L.M., Zhang, L.D., Chen, J.B., Huang, D.F., Zhang, Y.D.: Physiological analysis and transcriptome comparison of two muskmelon (Cucumis melo L.) cultivars in response to salt stress. - Genet. mol. Res. 15: 1-18, 2016.

Xie, C., Mao, X., Huang, J., Ding, Y., Wu, J., Dong, S., Wei, L.: KOBAS 2.0: a web server for annotation and identification of enriched pathways and diseases. - Nucl. Acids Res. 39: W316-W322, 2011.

Xu, J., Zhang, M., Liu, G., Yang, X., Hou, X.: Comparative transcriptome profiling of chilling stress responsiveness in grafted watermelon seedlings. - Plant Physiol. Biochem. 109: 561-570, 2016.

Yano, R., Nonaka, S., Ezura, H.: Melonet, D.B.: a grand RNASeq gene expression atlas in melon (Cucumis melo L.). - Plant Cell Physiol. 59: e4-e4, 2017.

Yue, C., Cao, H.L., Wang, L., Zhou, Y.H., Huang, Y.T., Hao, X.Y., Wang, X.C.: Effects of cold acclimation on sugar metabolism and sugar-related gene expression in tea plant during the winter season. - Plant mol. Biol. 88: 591-608, 2015.

Zago, E., Morsa, S., Dat, J.F., Alard, P., Ferrarini, A., Inzé, D., Van Breusegem, F.: Nitric oxide-and hydrogen peroxideresponsive gene regulation during cell death induction in tobacco. - Plant Physiol. 141: 404-411, 2006.

Zeng, F., Sun, F., Li, L., Liu, K., Zhan, Y.: Genome-scale transcriptome analysis in response to nitric oxide in birch cells: implications of the triterpene biosynthetic pathway. PloS ONE 9: 116-157, 2014.

Zhang, H., Wang, H., Yi, H., Zhai, W., Wang, G., Fu, Q.: Transcriptome profiling of Cucumis melo fruit development and ripening. - Hort. Res. 3: 16014, 2016.

Zhang, T., Zhao, X., Wang, W., Pan, Y., Huang, L., Liu, X., Fu, B.: Comparative transcriptome profiling of chilling stress responsiveness in two contrasting rice genotypes. - PloS ONE 7: e43274, 2012.

Zhao, R., Sheng, J., Shengnan, L.V., Zheng, Y.: Nitric oxide participates in the regulation of $L e C B F 1$ gene expression and improves cold tolerance in harvested tomato fruit. Postharvest Biol. Technol. 62: 121-126, 2011.

Zhu, J.H., Dong, C.H., Zhu, J.K.: Interplay between coldresponsive gene regulation, metabolism and RNA processing during plant cold acclimation. - Curr. Opin. Plant Biol. 10: 290-295, 2007. 